\title{
SET SIZE AND THE PART-WHOLE PRINCIPLE
}

\author{
MATTHEW W. PARKER \\ Centre for Philosophy of Natural and Social Science, \\ London School of Economics and Political Science
}

\begin{abstract}
Gödel argued that Cantor's notion of cardinal number was uniquely correct. More recent work has defended alternative "Euclidean" theories of set size, in which Cantor's Principle (two sets have the same size if and only if there is a one-to-one correspondence between them) is abandoned in favor of the Part-Whole Principle (if $A$ is a proper subset of $B$ then $A$ is smaller than $B)$. Here we see from simple examples, not that Euclidean theories of set size are wrong, nor merely that they are counterintuitive, but that they must be either very weak or in large part arbitrary and misleading. This limits their epistemic usefulness.
\end{abstract}

\$1. Introduction. On the standard Cantorian conception of cardinal number, equality of size is governed by what we will call...

Cantor's Principle (CP) Two sets have equally many elements if and only if there is a one-to-one correspondence between them.

Another principle that is at least naïvely appealing has been called (Mancosu, 2009)...

The Part-Whole Principle (PW) If $A$ is a proper subset of $B$, then $A$ is smaller than $B$.

This is a variant of Common Notion 5 from Euclid's Elements:

(CN5) The whole is greater than the part.

Hence we will call theories and assignments of set size Euclidean if they satisfy PW, ${ }^{1}$ despite the differences between PW and CN5 and doubts about the authorship of the Common Notions (Tannery, 1884; cf. Heath, 1956, 221).

It is well known that, for infinite sets, CP and PW are incompatible. Galileo (1939), for example, pointed out that the square numbers $(1,4,9,16, \ldots)$ can be matched one-to-one with the positive integers, though the squares also form a proper subset of the positive integers. (See Bunn, 1977; Parker, 2009; Mancosu, 2009 for references to earlier, related observations.) He therefore rejected the whole idea of relative size (larger and smaller) for infinite collections. Bolzano instead denied CP in favor of PW (Bolzano, 1950, 1973).

Today it is widely taken for granted that Cantor's theory is the only correct one and views like Galileo's and Bolzano's are just mistaken. Gödel argued explicitly for that position in 1947. But Gödel's argument relies heavily on certain intuitions while ignoring others. For example, he asks us to imagine transforming the physical objects in one set to be indistinguishable from those in another and infers without argument that such similar sets

Received: April 11, 2012.

1 This differs from some uses of 'Euclidean' in the related literature (cf. Mayberry, 2000; Benci et al., 2006). Note Mayberry's theory is finitistic and takes CP for granted; it is not particularly relevant to the present discussion. 
must have the same cardinality. Intuitively this seems right, but then so does PW. After all, if $A \subset B$, then $B$ contains all the elements of $A$ and more. Novice set theory students often resist $\mathrm{CP}$ because of such intuitions, and even great thinkers like Galileo, Leibniz, and Bolzano were unable to shake them (Parker, 2009; Leibniz, 2001). ${ }^{2} \mathrm{CP}$ and PW are both intuitive, so we cannot argue against one just by stirring sympathies for the other. Even if the intuitions supporting $\mathrm{CP}$ are stronger than those for PW, this is no reason to regard the latter as illegitimate, provided $\mathrm{PW}$ can be incorporated into a consistent theory of its own. ${ }^{3}$

In recent years, some authors have bucked the Cantorian hegemony to develop or defend Euclidean theories of set size (Katz, 1981; Benci \& Di Nasso, 2003a; Benci et al., 2006, 2007; Parker, 2009; Mancosu, 2009; Di Nasso \& Forti, 2010; Gwiazda, 2010). In fact, Benci \& Di Nasso (2003a) have shown that the existence of transfinite Euclidean set sizes is consistent with the standard set theory ZFC (first-order Zermelo-Frankel set theory with the Axiom of Choice). So provided ZFC is consistent ${ }^{4}$ and we do not insist on CP, there is no logical inconsistency in Euclidean sizes, even for infinite sets.

But our main question here is whether it is possible to have a really good Euclidean theory of set size, and my answer is, no, not really_not if that means it must be strong, general, well motivated, and informative. ${ }^{5}$ I will argue that any Euclidean theory strong and general enough to determine the sizes of certain simple, countably ${ }^{6}$ infinite sets must incorporate thoroughly arbitrary choices. Benci \& Di Nasso's (2003a) work shows that Euclidean set sizes are not determined by PW, even in combination with ZFC, the

2 Leibniz (2001, 181), for example, suggests that even God could not conceive a whole that is equal to its part. Strictly speaking, Galileo denies PW by concluding that infinite sets are incomparable, but only because he cannot see his way to rejecting either PW or CP for sets that are comparable. Leibniz (2001, 9), on the other hand, cannot accept even Galileo's compromise; a whole must always be greater than its part.

3 In fact, even Gödel $(1947,525)$ uses 'smallest' in a Euclidean sense within the very paper where he argues against such conceptions (as well as later revised editions, in his definition of "borelsets"). Such informal Euclidean uses of 'smallest' are commonplace in modern mathematics, but mainly in cases where the sets in question are nested, so that the "smallest" set is unambiguously determined by PW.

4 The consistency of ZFC has not been proven but is difficult to seriously doubt.

5 In response to helpful feedback from Sylvia Wenmackers, I will explain what I mean by these terms. By a general theory I mean one that applies to a broad domain or many domains, including, in particular, subsets of the whole numbers and countable point sets. By strong I mean logically strong - a theory that leaves little undecided, and in particular, one that determines the sizes of the simple sets discussed below. (This, incidentally, is a Euclidean notion: theory $A$ is stronger than theory $B$ if the theorems of $A$ properly include those of $B$. Again, I do not claim that Euclidean theories are useless, just very limited!) By well motivated I mean that the details of the theory - all of the particular sizes and size relations it assigns - are so assigned for some reason; they are not chosen arbitrarily. And finally, informative here means that the consequences of the theory (or concept) indicate something of interest that holds independently of the theory itself. The Cantorian power of a set, for example, tells us something about one-to-one correspondences and, in some contexts, Lebesgue measure. (Countable point sets always have Lebesgue measure zero.) Lebesgue measure in turn may tell us something about metric structure, the capacities of containers, or even chances. Arbitrarily assigned sizes, on the other hand, are not informative about anything.

6 There is no inconsistency or question begging involved in applying Cantorian concepts like countably infinite here. Our question is whether there are useful Euclidean conceptions of size in addition to Cantor's. Recent work on Euclidean theories, such as Benci, Di Nasso, and Forti's, employs a great deal of standard set-theoretical machinery, including Cantorian power. 
Continuum Hypothesis, finite additivity, and a number of nice algebraic properties. But furthermore, Euclidean sizes are not invariant under rigid transformations, and as we will see, this implies that, in some cases, Euclidean sizes must be as arbitrary as the choice of an origin or direction in a homogeneous space. Such sizes tell us very little about the sets they are supposed to characterize, and may even mislead us, as I will explain. And as it turns out, very simple, countable, and bounded sets violate rigid transformation invariance if PW holds. In fact, even the tiniest rotation of a bounded countable point set can result in an enormous change in set size. Moreover, even Euclidean relative sizes violate rigid transformation invariance: PW implies that for some equal-sized sets $A$ and $B$, the images $T A$ and $T B$ by a translation or rotation $T$ are not equal in size to each other. Here again, rigid transformation invariance is desirable because without it some size relations must be arbitrary, uninformative, and even misleading.

Thus I hope to provide a more cogent and detailed account than Gödel did of just what is "wrong with" Euclidean theories, without claiming that they are wrong and with as little reliance as possible on intuition. We might debate which definitions of set size best accord with our pre-theoretic intuitions, or which precise concept of size if any is picked out by our informal size-talk, but those are not my concerns here. What seems more important is the usefulness of size concepts. I want to make this emphatically clear: I am absolutely not arguing that Euclidean theories of set size fail to capture our pre-theoretic concept of size, nor that they are counterintuitive. (Of course they are, but so is any notion of transfinite size.) My point is that they are limited in their epistemic usefulness: either they fail to apply at all to many very simple sets or they are largely arbitrary, in which case they tell us very little about the sets they are meant to characterize. We will make certain basic assumptions about the properties of sizes, but what I am saying about such sizes does not depend on a conceptual analysis of size; I am making claims about their properties and their usefulness, not about whether we should call them sizes. Euclidean sizes are not only limited as measures of size; they are limited in their capacity to inform us about any properties of sets.

That is not to say that they are entirely useless. They may well have special applications to nonstandard probability theory (McCall \& Armstrong, 1989; Wenmackers \& Horsten, 2013; Gwiazda, 2010; Benci et al., 2013), the foundations of nonstandard analysis (Benci \& Di Nasso, 2003b) and number theory (Di Nasso, 2010). But anyone who has hoped for a revolutionary new Euclidean theory of set size comparable in breadth and value to Cantor's theory should be disappointed.

I also want to prevent another possible misunderstanding. It might be easy to misconstrue my argument along the following lines: "A rigid transformation maps a certain set $A$ onto a proper subset. Since this transformation is also a bijection, the image of $A$ is the same size as $A$, contrary to PW." Such an argument obviously presupposes the Cantorian notion of size, and therefore amounts to a petitio principii. But this is not my argument. I argue that Euclidean sizes violate not only $\mathrm{CP}$, but also other, weaker invariance principles specifically concerning rigid transformations, and that violating these weaker invariance principles implies arbitrariness. There is no petitio principii in this.

7 Thanks to an anonymous reviewer for pushing this point. This statement of the possible misconstrual (which the reviewer did not commit but warned me of) is closely paraphrased from the reviewer's remarks. 
A guiding principle behind the argument is this: transformations that preserve a lot of properties have more claim to preserve size than those that do not. ${ }^{8}$ Bijections in genenal do not preserve many properties; they just preserve Cantorian power ${ }^{9}$ and whatever else is determined by power. Homeomorphisms preserve power and topology, while rigid transformations preserve power, topology, and metric structure, that is, distance relations. Because rigid transformations preserve so much, I think they have a stronger claim to preserve size than bijections. I do not rely on this principle below; instead I try to show directly that the failure of rigid transformation invariance implies arbitrariness. But it may also be helpful to understand the justification of the gereral principle. It is just this: measures and relations are more useful if they inform us about the properties of the things they apply to. If two sets have all the same qualitative properties (properties other than the bare identities of their elements), and nontheless they are assigned different sizes, then that size difference does not tell us anything about the properties of the sets. So for the sake of epistemic utility, transformations that preserve all the properties and relations that we care about ought to prererve size as well. And there are many very standard contexts where metric structure and its implications are all we care about and all there is to care about. (This is true of topology too, but metric structure is stronger, so it ought to be slightly more striking and compelling that even metric structure does not determine Euclidean sizes.) The claim is not that metric structure is somehow implicated in the very concept of size; it is just that if sizes are not determined by other properties that we care about, then they are not very informative.

An important target of this critique is the theory of numerosities developed by Benci et al., (Benci \& Di Nasso, 2003a; Benci et al., 2006, 2007; Di Nasso, 2010; Blass et al., 2012). Numerosities are Euclidean set sizes with the algebraic and ordering properties of the ordinary whole numbers (i.e., those of a discretely ordered semi-ring). But I will not give a detailed account of numerosities, for the points to be made here are very general. Any Euclidean theory that determines the sizes of the examples below will be plagued with arbitrariness.

The examples are very simple and similar to well know cases where PW contradicts $\mathrm{CP}$, such as Galileo's paradox and Hilbert's hotel, so some may find them quite unsurprising. However, researchers developing Euclidean theories of size do not appear to have appreciated how simple and commonplace the examples are in which PW contradicts not only CP but also rigid transformation invariance. ${ }^{10}$ Nor do they seem to have taken in the consequences, namely that in such cases, Euclidean sizes are unavoidably arbitrary and potentially misleading.

8 An anonymous reviewer asked me to comment on this.

9 'Power' was Cantor's original term for what is now standardly called cardinal number. I am avoiding the word 'cardinal' and its derivatives because they suggest not only Cantor's technical concept but also the more general pre-theoretic notion of how many, the number of elements in a collection. I do not want to assume that Cantor's analysis of that notion in terms of one-to-one correspondence is uniquely correct, so I will stick with the less loaded term 'power.'

10 Di Nasso \& Forti (2010) note that numerosities are not preserved by rigid transformations because of the Banach-Tarski paradox, but as we will see, such a sophisticated result need not be invoked. Euclidean sizes violate rigid transformation invariance even for very simple, countable sets. As well, the argument from Banach-Tarski requires finite additivity, while in fact any Euclidean size assignment that applies to these simple sets must violate rigid transformation invariance, additive or not. 
§2. Notation, terminology, and background assumptions. We use $\mathbf{N}$ for the set of whole numbers (including zero), $\mathbf{Z}$ for the integers, $\mathbf{Z}^{+}$for the positive integers $(\mathbf{N} \backslash\{0\}$, where ' $\backslash$ ' is set difference), and $\mathbf{R}$ for the reals. 'P $(A)$ ' denotes the power set $\{B: B \subseteq A\}$, and $|A|$ the Cantorian power of $A$.

We will consider assignments of absolute sizes as well as assignments of size relations. The symbols ' $\triangleleft$,' ' $\unlhd$,' and ' $\triangleq$ ' will denote size relations between sets, so that ' $\unlhd$ ' for example should be read as 'is smaller than or equal in size to.' The familiar symbols ' $<$ ' and ' $\leq$ ' denote relations between absolute sizes rather than sets, and '=' denotes identity as usual. So to be precise,

\section{DEFINITION 2.1.}

(i) A size assignment is a size function or a size ordering.

(ii) A size function is a function [·] $: D \rightarrow X$ from a domain $D$ of sets into a linearly ordered set $\mathbf{X}=\langle X, \leq\rangle$.

(iii) If $[\cdot]$ is a size function, $[A]$ is called the absolute size of $A$.

(iv) A size function [.] is Euclidean on $D$ if for all $A, B \in D, A \subset B$ implies $[A]<[B]$.

(v) A size ordering (or size relation) $\unlhd$ is a reflexive, transitive relation (i.e., a preorder) on a domain $D$ of sets.

(vi) We write $A \triangleq B$ if $A \unlhd B$ and $B \unlhd A$.

(vii) We write $A \triangleleft B$ and $B \triangleright A$ if $A \unlhd B$ and it is not the case that $B \unlhd A$.

(viii) The relation $\triangleleft, \triangleright$, or $\triangleq$ that holds between sets $A$ and $B$, if any does, is called the relative size of $A$ to $B$.

(ix) A size ordering $\unlhd$ is Euclidean on $D$ if for all $A, B \in D, A \subset B$ implies $A \triangleleft B$.

Thus $<, \leq, \triangleleft, \unlhd$, and $\triangleq$ are all transitive, and $\triangleq$ is an equivalence relation. Note also that a size function defines a canonical size ordering: $A \unlhd B$ if and only if $[A] \leq[B]$. Conversely, if a size ordering is total it defines the Fregean size function $[A]=\{C \in D: C \triangleq A\}$, with $[A] \leq[B]$ if and only if $A \unlhd B$. So we can normally assume that $[A]<[B]$ if and only if $A \unlhd B$. Note also that an assignment is Euclidean on $D$ if PW applies to all proper subset/superset pairs in $D$. In that case, all such pairs have size relations, but this does not imply totality; we might for example have disjoint sets in $D$ that are not comparable at all.

Most of these stipulations are inspired by an intuitive analysis of size, but they can also be motivated by utility, since functions and relations with the above properties have special applications; or we can simply take them to demarcate the scope of the present inquiry. If we must abandon such basic assumptions to defend Euclidean sizes, that in itself illustrates how limiting such sizes are.

It is important for us to distinguish between size assignments, as just defined, and theories of size. Assignments are mathematical relations, and we impose no constraints on their complexity. They need not be constructive, definable, or recursively enumerable. In contrast, we will take a theory to be a deductively closed set of sentences or propositions generated by some recursive set of axioms and inference rules. We need not be completely explicit here; the important thing for us is that a theory, on this conception, is something we can state and work with. In the interest of usefulness, it must be somehow expressible, while an assignment need not be.

As noted above (footnote 9), we will avoid the word 'cardinal' and its derivatives, reverting to Cantor's earlier term 'power' for the equivalence classes induced by bijection. We will use 'count' for the more general and imprecise notion of number of elements, 
or how many, as distinguished from ordinal positions like seventh and nonintegral magnitudes like $81 / 2$ or $-\pi$. Some arguments here assume a property central to notions of count, namely...

Discreteness If $A \triangleleft B$ then $A \cup\{x\} \unlhd B$.

This says that two sets cannot differ in $\unlhd$-size by less than a whole element. It has particular interest because of the ancient project of understanding the relation of count to geometric measure and that of individual points to the continuum. Furthermore, for sets that are not vague or fuzzy and do not differ by a part of an object (as in the case of $61 / 2$ apples compared to 6 apples), sizes that differ by less than a whole element would be misleading. They would suggest that we could make the sets equal by adding or removing a part of an object, or by including an object more definitely. But neither holds for the discrete sets of numbers and points we will consider; they contain only whole elements and contain them wholly. So we have good reasons to assume Discreteness, but most arguments here do not require it.

We will need a few more expressions, concepts, and postulates, but these will be introduced as they come to bear.

§3. The trouble with arbitrariness. Here I want to say a little about what I mean by arbitrariness and why it is undesirable, and head off a couple more possible misunderstandings.

'Arbitrary' can mean many things, but the main thing I mean by it here is that Euclidean size assignments are unmotivated in many of their specific details. Particular sizes could be chosen differently without any significant loss of utility or elegance in the system of sizes. One might object that, in connection with Euclidean theories, arbitrariness is motivated, since it is better to have a partly arbitrary Euclidean theory than none at all. ${ }^{11}$ But this confuses the issue. The first question is not whether arbitrariness itself is motivated but whether the particular details of a given Euclidean size assignment are well motivated. If not all of them are, that is what I call arbitrariness. Whether the benefits of a partly unmotivated theory outweigh its detractions is another question. Perhaps such a theory is useful for some purposes, but it is also limited and, as I will argue shortly, misleading.

Another possible objection is that the Euclidean theories on offer do not determine sizes arbitrarily; they leave sizes indeterminate where well motivated principles do not decide them, and this is just what they should do. ${ }^{12}$ Indeed, the theories of Benci et al. and others do not determine the sizes of all sets or even all sets of whole numbers. However, those theories are about Euclidean assignments that are total over some broad class of sets. In most of their papers, Benci et al. define the notion of numerosities so that they must be defined over a broad domain such as the entire power set of $\mathbf{N}$ (Benci \& Di Nasso, 2003a) or the class of all sets of ordinals (Benci et al., 2006). Even where totality is not built into the definition (e.g., Di Nasso \& Forti, 2010), numerosities are elaborately constructed from selective ultrafilters (special nonconstructive collections of sets) precisely to ensure totality. So the theory of Benci et al. may not decide sizes arbitrarily, but the assignments that the theory describes do. My main claim is that Euclidean theories and assignments must be either arbitrary or too weak to decide the relative sizes of some very simple sets. The theory of numerosities suffers more from the latter limitation, and numerosities themselves from

11 One reader of an earlier draft made such a remark.

12 This is another comment made by a reader. 
the former. But as we will see, even Euclidean theories that do not determine all set sizes must be somewhat arbitrary.

But what, you may ask, is so bad about arbitrariness? If there is no good reason to say that $A$ is not smaller than $B$, for example, then why not just stipulate that it is? The problem with this is that it is misleading. The assignment of different sizes to two sets gives the impression that there is some significant dissimilarity between them, but as we will see, if we assign Euclidean sizes to the sets described below, we must sometimes assign different sizes to sets that are as similar as can be. Such a size assignment would misguide us into seeing differences where there are none. A nonarbitrary size assignment would be one that reflects facts or theoretical consequences that hold independently of the notion of size employed. ${ }^{13}$ (In Cantor's theory, for example, sizes always reflect the existence or nonexistence of one-to-one correspondences.) If instead some sizes must be chosen arbitrarily, we face the problem of extricating the meaningful results about sizethose that reflect antecedent facts-from those that are merely consequences of our free choice.

One might object that we must always contend with arbitrary, conventional elements in our theories, since our choice of language always involves some arbitrariness. But the unavoidable arbitrariness of language lies mainly in the choice of symbols used rather than the measures or concepts employed. Where the concepts themselves are also somewhat arbitrary (as with, say, the folk distinction between frogs and toads), this limits their usefulness and scientific interest, just as I claim it does for Euclidean sizes.

Now to the examples.

§4. Absolute translation invariance. Consider the sets $\mathbf{N}=\{0,1,2, \ldots\}$ and $\mathbf{Z}^{+}=$ $\{1,2,3, \ldots\}=\mathbf{N} \backslash\{0\}$. According to $\mathbf{P W}, \mathbf{N}$ is larger than $\mathbf{Z}^{+}$. Yet $\mathbf{Z}^{+}$is merely a translation of $\mathbf{N}$ by one unit. So already we have a violation of translation invariance for any Euclidean size assignment that applies to these sets.

Intuitively, a translation is a transformation that preserves distances as well as directions. We can conveniently define it as follows:

Definition 4.1. A translation on a metric space ${ }^{14}\langle S, d\rangle$ is a map $T: S \rightarrow S$ such that for all $p, q \in S, d(p, q)=d(T p, T q)$ and $d(p, T p)=d(q, T q){ }^{15}$

What we saw in the preceding paragraph is that Euclidean sizes on the sets of whole numbers violate

Absolute Translation Invariance (ATI) If $T$ is a translation on a metric space $\langle S, d\rangle$ and $A \subseteq S$ then $T A \triangleq A$.

13 This suggests a degree of realism, and indeed, for the purposes of this paper, I take it for granted that there are some objective logical facts that are relevant to our discussion-in particular, facts about which propositions do and do not follow from ZFC and other theories, given the standard ways of interpreting those theories. I do not suppose, as Gödel did, that there is any objective fact about what the size of an infinite set consists in, or whether set sizes satisfy CP or PW, for I think we are free to employ nonstandard and even unnatural notions of size if we wish. However, I do regard it as a matter of fact that the informativeness of Euclidean size assignments is limited in certain ways.

14 A metric space is just a set equipped with a measure of distance. The precise definition can be found in any introductory analysis textbook or appropriate Internet resource.

15 This is not, as far as I know, a standard definition of 'translation,' but it is simple and captures the right cases for our purposes. 
For if we define $d(n, m)$ as the absolute value $|n-m|$, then $\langle\mathbf{N}, d\rangle$ is a metric space, $T n=n+1$ is a translation, and $\mathbf{Z}^{+}=T \mathbf{N}$. But according to PW, $T \mathbf{N} \triangleleft \mathbf{N}$. So Euclidean sizes violate ATI for very simple sets in very ordinary contexts.

It might be tempting to object that invariance principles like ATI are all too Cantorian. If we are going to evaluate the competing principles $\mathrm{CP}$ and $\mathrm{PW}$, it might be argued, we should not assume that certain mappings must preserve size, as that is too prejudiced towards $\mathrm{CP} .{ }^{16}$ But here we are assuming no such thing. We are merely noting that besides contradicting CP, PW also contradicts weaker principles like ATI, and as we will see, this implies arbitrariness. Even if we are prepared to drop CP, it would be useful if the size of a set were not arbitrarily assigned but indicated some significant property of that set. But as we will see, if ATI fails this is not the case; sets as similar as can be (and some not related by inclusion) must differ in size, and this in itself is somewhat arbitrary. Furthermore, the failure of ATI implies that the size relations between certain spatially unrelated sets must be arbitrary; they can't have any motivation. So the failure of ATI might seem natural to some advocates of $\mathrm{PW}$, but it is nonetheless very limiting.

The example of $\mathbf{N}$ and $\mathbf{Z}^{+}$is a simple, concrete illustration of the conflict between PW and ATI, but that conflict is not restricted to whole numbers; it easily generalizes to any space where some translation has an infinite forward orbit.

Definition 4.2. A forward orbit of a map $T: D \rightarrow R$ is a set $\left\{T^{n} p: n \in U\right\}$ where $p \in D$ and either $U=\mathbf{N}$ or $U=\{0,1, \ldots, m\}$ for some $m \in \mathbf{N}$.

We make use of the fact that if a map has an infinite forward orbit (i.e., there are infinitely many distinct $T^{n} p$ ) applying the map to that orbit yields a proper subset of the orbit:

LEMmA 4.3. If $P$ is an infinite forward orbit of a map $T$ then $T^{n} P \subset P$ for all $n \in \mathbf{Z}^{+}$.

Proof. Let $P=\left\{T^{n} p: n \in \mathbf{N}\right\}$ be infinite for some $p \in \operatorname{dom}(T)$. If $q \in T^{n} P$ then $q=T^{n}\left(T^{m} p\right)$ for some $m \in \mathbf{N}$. In that case, $q=T^{n+m} p \in P$. So $T^{n} P \subseteq P$. Now we show that $p \notin T^{n} P$. For suppose otherwise: Then $p=T^{n}\left(T^{m} p\right)$ for some $m$, and hence $p=T^{n+m} p=T^{k(n+m)} p$ for each $k \in \mathbf{N}$. So for each $q \in P, q=T^{j} p=T^{j \bmod (n+m)} p$ for some $j$. But there are only $n+m$ different values of $j \bmod (n+m)$ and hence only $n+m$ distinct elements of $P$. This contradicts the assumption that $P$ is infinite, so in fact $p \notin T^{n} P$ and thus $P$ is not included in $T^{n} P$.

This brings us to a simple but crucial lemma for this paper: no map with an infinite forward orbit preserves Euclidean size. ${ }^{17}$

LEMmA 4.4. If $T: D \rightarrow R$ has an infinite forward orbit $P$ and $\unlhd$ is a size ordering that is Euclidean (but not necessarily total) on $\mathcal{P}(D)$, then $T P \triangleleft P$.

Proof. By Lemma 4.3, $T P \subset P$. Since $P$ is infinite, each $p \in P$ has an image $T p$. So $P \subseteq D$, and hence $T P, P \in \mathcal{P}(D)$. By Euclideanism, $T P \triangleleft P$.

Applying this lemma to translations, we see that Euclidean sizes violate ATI:

REMARK 4.5. If $T$ is a translation on $\langle S, d\rangle$ with an infinite forward orbit and $\unlhd$ is Euclidean on $\mathcal{P}(S)$, then $\unlhd$ violates $A T I$.

Proof. Immediate from Lemma 4.4

16 A reader of an earlier draft posed this argument.

17 Essentially the same observation was made by Benci et al. (2007, §3.1). 


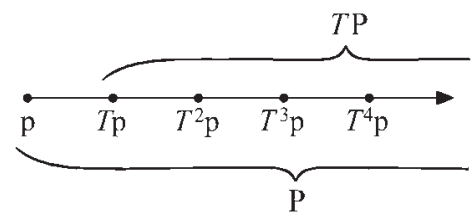

Fig. 1. Here $T P=\left\{T^{n} p: n \in \mathbf{N}\right\}$ not only has a one-to-one correspondence with its proper superset $P$, but is also a rigid translation of $P$. Yet, given $\mathrm{PW}, T P$ is smaller.

Specifically, if $P=\left\{T^{n} p: n \in \mathbf{N}\right\}$ is an infinite forward orbit of a translation $T$, then $T P \subset P$ (see Figure 1). So if PW holds for these two sets, then $T P \triangleleft P$, and thus ATI fails. ${ }^{18}$ And translations with infinite forward orbits are common; they always exist in Euclidean spaces (in the usual geometric sense of Euclidean-see Definition 7.1) as well as many other spaces. So in many ordinary contexts, PW contradicts ATI.

This is a trivial result, but its implications are worth considering. In particular it means that under Euclidean size assignments, sets that are about as similar as any two sets can be must nonetheless differ in size. ${ }^{19} P$ and $T P$ may be exactly alike in both their structure (the relations between the elements of a set-I will say more about this below) and the qualitative properties of their elements (e.g., they may be geometric points with no distinctive qualities at all). Yet by PW they must be unequal in size.

This is not only counterintuitive but impractical. One of the very useful features of our usual notions of size is that they meaningfully classify nonidentical things as equal. One can have exactly as many apples as oranges, however incomparable the individual fruits. But Euclidean sizes are very limited in this respect. PW tells us that certain sets are not equal in size, but it does not tell us which ones are, and it leaves many cases

18 This also illustrates a fundamental conflict between two of the Common Notions in the Elements. Common Notion 4 is translated by Heath (1956) as,

(CN4) Things coinciding with one another are equal to one another.

(Heath also suggests, and Benci et al. in 2007 prefer, 'applying onto' in place of 'coinciding with.') If the sense of 'coinciding with' (or 'applying onto') is the sense in which, for example, one line segment coincides with another of the same length, then surely the sets $P$ and $T P$ coincide as much as any two sets or figures, so by $\mathrm{CN} 4$ they must be equal. Yet $T P$ is a proper part of $P$, so by CN5, $P$ is larger. This inconsistency does not depend on whether we interpret 'equal' and 'greater' in terms of count, geometric measure, or some other notion of size. As long as we take $\mathrm{CN} 4$ and $\mathrm{CN} 5$ to concern the same notion of size, examples like $P$ and $T P$ show that they are mutually inconsistent. This is also obvious for unbounded figures like rays and half-planes: if point $b$ is between $a$ and $c$, then ray $b c$ is a proper part of ray $a c$, and yet the two rays surely "coincide with" each other. But below we will see examples of bounded point sets for which CN4 and CN5 are inconsistent. (Benci et al. (2007) claim that their numerosities satisfy both CN4 and CN5, but this is because they interpret 'applying onto' as 'related by an isometry,' and they define an isometry as a numerosity-preserving transformation. This makes CN4 trivial and empty.)

19 Some sets might perhaps be considered more similar than $P$ and $T P$. For example, let $A=$ $\{(0,1),(0,2),(0,3), \ldots\} \subseteq \mathbf{R}^{2}$. Let $A^{+}=A \cup\{(1,0)\}$ and $A^{-}=A \cup\{(-1,0)\}$. Then $A^{+}$and $A^{-}$are mirror images of each other, and they have almost all of their elements in common. And under some Euclidean size assignments (the numerosities of Benci et al., in particular), such sets do have the same size. But this is little consolation for the fact that sets as similar as $P$ and $T P$ must differ in size, and so, as we will see, must other, equally similar but disjoint sets. 
undetermined. ${ }^{20}$ And since it implies that qualitatively identical sets like $P$ and $T P$ must differ in size, the fact that two sets are not equal in size under a given Euclidean assignment has very little significance. No amount of similarity is sufficient for equal size, so unequal size tells us nothing about how alike or unalike the sets in question are.

One might at first suppose that unequal size tells us that two sets are related by proper inclusion, or perhaps that they are either related by proper inclusion or qualitatively different. But no, under any sufficiently strong Euclidean assignment, some sets that are exactly alike and disjoint must not be equal. That is,

REMARK 4.6. If $T: S \rightarrow S$ is a translation with an infinite forward orbit and $\unlhd$ is a Euclidean size ordering on $\mathcal{P}(S)$, then there is a countably infinite set $Q$ such that $Q \cap T Q=\varnothing$ and it is not the case that $Q \triangleq T Q$.

Proof. Since $T$ has an infinite forward orbit, so does $T^{2}$. So let $Q=\left\{p, T^{2} p, T^{4} p, \ldots\right\}$ $=\left\{T^{2 n} p: n \in \mathbf{N}\right\}$ be countably infinite (see Figure 2). Then by Lemma $4.4, T^{2} Q=$ $\left\{T^{2} p, T^{4} p, T^{6} p, \ldots\right\} \triangleleft Q$. Now notice that $T Q=\left\{T p, T^{3} p, T^{5} p, \ldots\right\}$ is disjoint from both $Q$ and $T^{2} Q$. But it cannot be equal to both, for if $Q \triangleq T Q$ and $T Q \triangleq T^{2} Q$ then by transitivity $Q \triangleq T^{2} Q$, contradicting Lemma 4.4. So either it is not the case that $Q \triangleq T Q$ or it is not the case that $T Q \triangleq T^{2} Q$, and in either case the remark holds. ${ }^{21}$

So given PW, some sets that are about as alike as can be and not related by inclusion still do not have the same size. Thus the fact that two sets are not alike in Euclidean size tells us very little about them. Granted, it does tell us something, namely that neither set includes the other. And it might tell us more given a Euclidean size assignment with particular properties. (For example, if the assignment is finitely additive, then the fact that $A$ is not equal in size to $B$ implies that $A$ and $B$ cannot be partitioned into disjoint sets $A_{1} \cup A_{2}=A$ and $B_{1} \cup B_{2}=B$ such that $A_{1} \triangleq B_{1}$ and $A_{2} \triangleq B_{2}$.) But the fact that sets as alike as $Q$ and $T Q$ are not equal in size even in cases where neither includes the other illustrates how very little nonequality means under Euclidean sizes.

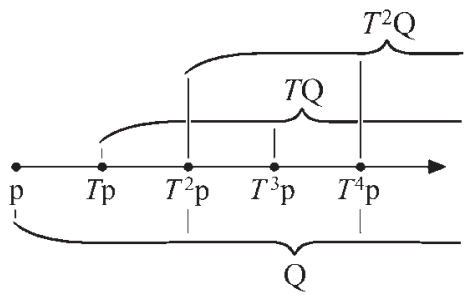

Fig. 2. $T^{2} Q=\left\{T^{2} p, T^{4} p, T^{6} p, \ldots\right\}$ is a proper subset of $Q=\left\{p, T^{2} p, T^{4} p, \ldots\right\}$, while $T Q=\left\{T p, T^{3} p, T^{5} p, \ldots\right\}$ is disjoint from both. And given PW, it cannot be equal in size to both.

20 Benci et al. (2007) also concern themselves with bases for equality, or as they put it, the search for interesting "isometries," which they define as transformations that preserve numerosity. They point out that certain product principles imply certain limited invariance properties (Benci et al., 2006, 2007). However, as they also acknowledge, their product principles are problematic (Benci et al., 2006, 45; 2007, 48), and PW imposes severe restrictions on size-preserving maps, since, as our Lemma 4.4 states, no map with an infinite forward orbit preserves Euclidean sizes (Benci et al., 2007, §3.1). So the prospects for interesting isometries (in this sense) are dim.

21 The example of $Q, T Q$, and $T^{2} Q$ is closely related to the coin flipping examples in Williamson's (2007) critique of nonstandard probabilities as well as the case of Even and Odd discussed by Benci et al. (see below). 
This begins to show why the failure of translation invariance implies arbitrariness. If sets that are entirely alike except for where they happen to be located have different sizes, then these sizes are hardly well motivated. But we can make this sharper in certain contexts. In mathematics and physics, we often want to consider spaces that have no designated coordinate system and no privileged positions. Let us call these homogeneous coordinatefree spaces. In such spaces, two sets like $Q$ and $T Q$ or $P$ and $T P$ are very much on an equal footing. The positions occupied by $P$ under one coordinate system are occupied by $T P$ under another, and in particular under a coordinate system that is just a translation of the first. Hence, assigning a particular size to $P$ rather than $T P$ is as arbitrary as a choice between two coordinate systems that differ only with respect to which point is taken as the origin. And in a homogenous coordinate-free space, there is by hypothesis no reason to choose one point rather than another as the origin.

But we need to be careful here. We noted above that, given a size ordering $\unlhd$, we can always adopt a Fregean system of absolute sizes in which $[A]=\{B: B \triangleq A\}$. Even in a homogeneous coordinate-free space, we might regard this as a privileged assignment. (It would be very odd to let $[A]=\{B: B \triangleq C\}$ where $C$ is not the same size as $A$.) So there does seem to be a nonarbitrary assignment of absolute sizes, provided we already have nonarbitrary relative sizes. But suppose we start with some pre-existing linearly ordered set $\mathbf{X}=\langle X, \leq\rangle$ of "sizes," such as the hyper-integers (special equivalence classes of infinite sequences of integers) that Benci et al. use as numerosities. Then we come across point sets like $P$ and $T P$ in a space that is not related to $\mathbf{X}$ in any special way. Now we have a problem: for any size $x \in X$ we assign to $P$, we could just as well assign $x$ instead to the translation $T P$ or $T^{217} P$. How, for example, could we ever assign hyper-integers to point sets in physical space? Arguably, this is a problem even if the background space has a designated coordinate system, but in cases where it does not, the impossibility of a well motivated choice is clear: since the position held by $P$ in one coordinate system is the same position held by $T P$ in another that is no more privileged, the assignment of $x$ to $P$ rather than $T P$ would be as arbitrary as the choice of an origin in a homogeneous coordinate-free space.

Even if we do not start with some designated linearly ordered set of absolute sizes, there is a more general underlying problem. The failure of ATI means there is no nonarbitrary way of comparing certain isometric sets that live in different coordinate-free spaces. Isometric sets are those related by a distance-preserving transformation such as a translation:

DeFINITION 4.7. Two sets $A$ and $A^{\prime}$ are isometric to each other, relative to metrics $d$ and $d^{\prime}$, if there is a bijection $T$ from $A$ to $A^{\prime}$ such that for all $p, q \in A, d(p, q)=d^{\prime}(T p, T q)$.

Now suppose ATI fails on some homogeneous coordinate-free metric space $\mathbf{S}=\langle S, d\rangle$. Then there are two sets $A, T A \subseteq S$ that are related by a translation, and therefore isometric, but not equal in size. Suppose also that we have another set $B$ in some other space $\mathbf{S}^{\prime}=$ $\left\langle S^{\prime}, d^{\prime}\right\rangle$ spatially unrelated to $\mathbf{S}$, and $B$ is isometric to $A$. Then of course $B$ is isometric to $T A$ as well. Assume also that the points themselves have no distinct qualitative properties. They are just points. Now how could we assign a size relation between $B$ and $A$ ? If we say that $B \triangleq A$, then we cannot also have $B \triangleq T A$. But we might just as well have said that $B \triangleq T A$ instead, since there is no difference between $A$ and $T A$ except their positions, and $\mathbf{S}$ has no privileged positions. Either stipulation would be arbitrary. Nor can we say without arbitrariness that $B \triangleleft A$ or that $A \triangleleft B$, because $A$ and $B$ have exactly the same 
metric structure and qualitative properties, and by hypothesis they have no special relation to each other. So if we assign any size relation at all between $B$ and $A$, it must be arbitrary.

If ATI held, we would not have the same problem. Then we might assign sizes based on structural features of some kind. Sets like $A, T A$, and $B$, alike in every respect except location, could all have the same size. At least then we would have some nonarbitrary way of assigning size relations to them. But if ATI fails, this is impossible.

So here the conclusion is definite: the failure of ATI implies arbitrariness. If ATI fails on a metric space $\mathbf{S}$, then there are sets in $\mathbf{S}$ and another unrelated space $\mathbf{S}^{\prime}$ that can only have arbitrary size relations if any.

\$5. ATI, number sets, and structure. For sets of whole numbers we cannot quite make the same argument. We cannot claim that there is no qualitative difference between a set of whole numbers and a translation thereof, such as $\mathbf{N}$ and $\mathbf{Z}^{+}$, or Even $=$ $\{0,2,4, \ldots\}=\{2 n: n \in \mathbf{N}\}$ and Odd $=\{1,3,5, \ldots\}=\{2 n+1: n \in \mathbf{N}\}$. The set $\mathbf{N}$, for example, begins with 0 , while $\mathbf{Z}^{+}$begins with 1 , and, at least on one view, there are qualitative differences between 0 and 1, and between any two distinct whole numbers. Some structuralists might claim that whole numbers have no distinctive intrinsic properties; they are just positions in a sequence. But numbers can also be understood as properties of sets, and the property of having zero elements is very different from that of having one. So in this respect there is a qualitative difference between $\mathbf{N}$ and $\mathbf{Z}^{+}$, and likewise between any two sets of whole numbers. Hence, the failure of ATI for number sets does not directly imply that their sizes are arbitrary.

But when we evaluate the sizes of number sets, it is rarely if ever the particular intrinsic properties of the individual numbers that we wish to characterize. As noted above, part of the value of notions of size or count is that they enable us to classify sets as equal in cases where they do not contain the same or qualitatively identical elements. So set sizes that depend on the intrinsic properties of individual elements are not very helpful.

Still, a useful notion of size would be one that tells us something about the sets it measures. If it does not tell us about the intrinsic natures of a set's individual elements, then presumably it should tell us something about the relations between the elements, or the structure of the set. Structure is widely regarded as important; some philosophers have even suggested that structure is what mathematics is all about (e.g., Resnik, 1997). I do not want to overemphasize this; there is a risk of being drawn into a conceptual analysis of structure or a debate over its relevance to concepts of size, and I do not want to do that here. But I do want to point out that Euclidean sizes of number sets are not informative about certain salient kinds of structure, and it is not clear what else they might be informative about.

We might say that two sets have the same structure if there is a bijection between them that preserves the relations between elements. So sets with the same structure have the same power, but not just that; their elements also stand in the same relations. The problem with this definition is, only the identity map preserves all relations between elements. Given a bijection between distinct sets, we can always concoct some relation that it does not preserve. So if we want to talk about structure, it has to be relative to some particular class of relations, and preferably relations of particular interest.

Translations preserve distance, and we have seen that, given PW, they do not preserve size, for PW implies that $\mathbf{N} \triangleright \mathbf{Z}^{+}$. So if distance (or arithmetic difference) is one of those relations we care about, then Euclidean sizes are not very informative about structure. The fact that two sets have different sizes under a given Euclidean assignment does not tell us 
that they have different metric structures. This does not prove that Euclidean sizes must be completely arbitrary for number sets, but if metric structure is something we care about, it is a limitation.

But perhaps metric structure is not the most important kind here. Even if we agree that we would like set sizes to tell us something about the relations between elements, there is no strong reason that they must tell us about distance relations. We are especially concerned with metric structure in this paper because in some important contexts it is the only structure on offer, so there, if Euclidean sizes do not tell us about metric structure, they do not tell us much about anything. But when we are talking about number sets, we might be more concerned with algebraic structure. So perhaps mappings that preserve addition and multiplication, that is, those that preserve the three-place relations $+(a, b, c) \Leftrightarrow a+$ $b=c$ and $\cdot(a, b, c) \Leftrightarrow a \cdot b=c$, might preserve Euclidean size. But in fact, no, for consider Odd $=\{2 n+1: n \in \mathbf{N}\}$ and $\mathbf{O d d}^{2}=\left\{n^{2}: n \in \mathbf{O d d}\right\}$. In either of these sets, no element is a sum of two others, so any mapping between them preserves addition vacuously. And the mapping $f(a)=a^{2}$ preserves multiplication, so Odd and Odd ${ }^{2}$ have the same algebraic structure. But $\mathbf{O d d}^{2} \subset$ Odd, so by PW, Odd ${ }^{2}$ is smaller.

Thus, if there is some important sort of structure that Euclidean sizes are informative about, it is not obvious what it is. For point sets we reached a stronger conclusion: In certain cases, the assignment of Euclidean sizes must be utterly arbitrary. But even for number sets, the epistemic usefulness of Euclidean sizes is limited.

§6. Relational translation invariance. Though translations often do not preserve Euclidean size, one might hope that they would at least preserve the Euclidean size relations between sets. That is, we would like...

Relational Translation Invariance (RTI) If $T: S \rightarrow S$ is a translation on a metric space $\langle S, d\rangle$ and $A, B \subseteq S$ then $A \unlhd B$ if and only if $T A \unlhd T B$.

But no, even this fails under mild conditions. For a concrete example, consider the sets Even $=\{0,2,4, \ldots\}$, Odd $=\{1,3,5, \ldots\}$, and Even $+2=\{n+2: n \in$ Even $\}=$ $\{2,4,6, \ldots\}$. Assuming PW, Even $\triangleright$ Even +2 . But is Odd the same size as Even or as Even +2 ? Or neither? Any choice among these is arbitrary. Why for example should Even be equal in size to its translation Odd while Odd is not equal to its translation Even +2 ?

To put the point more precisely, any such choice violates RTI, assuming PW and Discreteness. For given Discreteness we cannot have Even $\triangleright$ Odd $\triangleright$ Even +2 . So either Even $\unlhd$ Odd or Odd $\unlhd$ Even +2 . Either way, RTI implies that Even $\unlhd$ Odd $\unlhd$ Even + 2, contradicting PW.

This generalizes straightforwardly to any invertible translation with an infinite forward orbit:

REMARK 6.1. If $T: S \rightarrow S$ is an invertible translation with an infinite forward orbit and $\unlhd$ is a total Euclidean size ordering on $\mathcal{P}(S)$ satisfying Discreteness, then $\unlhd$ violates RTI.

Proof. Let $\left\{T^{n} p: n \in \mathbf{N}\right\}$ be an infinite forward orbit of $T$ and let $Q=\left\{T^{2 n} p: n \in \mathbf{N}\right\}$ as above. By PW, $T^{2} Q \triangleleft Q$. By Discreteness, we cannot have $Q \triangleright T Q \triangleright T^{2} Q$. So by totality, $T Q \unlhd T^{2} Q$ or $Q \unlhd T Q$. In the former case, RTI implies that $Q \unlhd T Q$, and in the latter, that $T Q \unlhd T^{2} Q$. Either way, $Q \unlhd T Q \unlhd T^{2} Q$, contradicting PW.

Here we have assumed Discreteness and totality. As argued in §2, it would be misleading to drop Discreteness. We will discuss dropping totality in $§ 9$. But we will soon see that RTI 
also fails without Discreteness or totality, under other mild conditions. (The incompatibility of PW and RTI is robust in something like the sense of Wimsatt (1981): it manifests under different, independent conditions - and weak ones to boot.)

Violations of RTI are misleading. Suppose, for example, we have Even $\triangleq$ Odd but Odd $\triangleright$ Even + 2. This would suggest that there is some special relation between the former two sets that does not hold between the latter. But there is not. Even and Odd are related in exactly the same way as Odd and Even +2 , namely by the translation $T n=n+1$. Of course, one can define relations that hold between Even and Odd and do not hold between Odd and Even +2 , but by the same token, lots of relations hold between the latter two and not the former. The point is, there is no particularly important or size-like relation that holds of the former two and not the latter-nothing to warrant assigning one pair of sets the same size but not the other pair. We could just as well stipulate that Even $\triangleright$ Odd $\triangleq$ Even +2 , and adopting either ordering over the other would just obscure the fact that neither is privileged.

In fact, Benci \& Di Nasso (2003a) have shown that the existence of numerosities satisfying either one of these stipulations, Even $\triangleright$ Odd $\triangleq$ Even $+\mathbf{2}$ or Even $\triangleq$ Odd $\triangleright$ Even +2 , is consistent with ZFC. (The existence of such numerosities is implied by the existence of selective ultrafilters on $\mathbf{N}$, which is itself independent of ZFC but implied by the Continuum Hypothesis.) So even ZFC, PW, the strong algebraic and ordering properties of numerosities, and the Continuum Hypothesis, all taken together, do not resolve basic questions like whether Even $\triangleq$ Odd.

Benci and Di Nasso suggest that such indeterminacy can be overcome by introducing further postulates, such as $n\left[n \mathbf{Z}^{+}\right]=\left[\mathbf{Z}^{+}\right]$, that is, that the sum of $n$ terms each equal to $[\{n, 2 n, 3 n, \ldots\}]$ is $\left[\mathbf{Z}^{+}\right]$. Given the algebraic properties of numerosities, this implies that Even $\triangleright$ Odd $\triangleq$ Even $+2{ }^{22}$ They also propose a similar postulate concerning powers. But there is no indication that such postulates can resolve all of the indeterminacy of numerosities, only indications to the contrary. All known existence proofs for Euclidean sizes on infinite sets are nonconstructive, since they appeal to the Axiom of Choice or related axioms. Those for numerosities go further, making use of nonprincipal ultrafilters. Pincus \& Solovay (1977) have shown that there are models of ZFC in which no such ultrafilter is definable. So prima facie, it may be impossible to define a unique total Euclidean size assignment on $\mathcal{P}(\mathbf{N})$, no matter how many postulates we adopt. A hefty burden of proof would rest on anyone claiming otherwise.

In any case, postulates like Benci and Di Nasso's cannot resolve the arbitrariness of Euclidean sizes unless the postulates themselves are nonarbitrary. And what is there to recommend a postulate like $n\left[n \mathbf{Z}^{+}\right]=\left[\mathbf{Z}^{+}\right]$? It may look natural if we are particularly interested in $\mathbf{Z}^{+}$, but one could just as reasonably stipulate that $n[n \mathbf{N}]=[\mathbf{N}]$ instead, and then we would have Even $\triangleq$ Odd $\triangleright$ Even $+\mathbf{2}$.

The underlying difficulty is that all of these assignments violate RTI. If Even and Odd are equal in size, then Odd and Even $+\mathbf{2}$ are not, and vice versa, assuming PW. So the size relation between two sets depends not only on their structural properties and external relations to each other but on where in particular they happen to be on the number line. Thus the choice between stipulations like Even $\triangleq$ Odd and Odd $\triangleq$ Even +2 , or between $n\left[n \mathbf{Z}^{+}\right]=\left[\mathbf{Z}^{+}\right]$and $n[n \mathbf{N}]=[\mathbf{N}]$, is as arbitrary as the choice of a favorite position on the number line.

22 Benci and Di Nasso use 'Even' to denote our set Even $+\mathbf{2}=\{2,4,6, \ldots\}$. On that convention, their postulate implies that Even $\triangleq$ Odd, which perhaps makes it seem more natural and egalitarian, but this is just a superficial artifact of their notation. 
And even if one could eliminate the indeterminacy of Euclidean size assignments on $\mathcal{P}(\mathbf{N})$, there would remain many other indeterminacies in other contexts. As with ATI, the failure of RTI is even more worrying for point sets than for number sets. We might concede the possibility that 'Even $\triangleq$ Odd $\triangleright$ Even +2 ' ' is a better stipulation than 'Even $\triangleright$ Odd $\triangleq$ Even +2 ' for some reason having to do with the distinct properties of the numbers involved. But for our point sets $Q, T Q$, and $T^{2} Q$, in the context of a space with no privileged origin, there is no such possibility. To stipulate that, say, $Q \triangleq T Q \triangleright T^{2} Q$ would be indisputably arbitrary, because the sets $Q$ and $T Q$ take the same positions in one coordinate system as $T Q$ and $T^{2} Q$ take in another-one that differs from the first only in the placement of its origin-and there is nothing intrinsic in the points themselves to distinguish the relation of $Q$ to $T Q$ from that of $T Q$ to $T^{2} Q$.

Now let us see a violation of RTI without Discreteness or totality. It is more than enough to assume that $\mathbf{N}$ is the same size as its reflection $-\mathbf{N}=\{0,-1,-2, \ldots\}$. Then if $T i=$ $i+1$ for all $i \in \mathbf{Z}$, PW implies that $T \mathbf{N} \triangleleft \mathbf{N}$ and $-\mathbf{N} \triangleleft T(-\mathbf{N})=\{1,0,-1, \ldots\}$. By transitivity, $T \mathbf{N} \triangleleft T(-\mathbf{N})$, and since $\mathbf{N} \triangleq-\mathbf{N}$, this contradicts RTI.

In fact, we can get a more general result with only a very weak assumption about orbits of translations.

DEFINITION 6.2. A backward orbit of an invertible map $T$ is a forward orbit of its inverse $T^{-1}$ (where $T^{-1} p$ is the unique point $q$ such that $T q=p$ ).

Backward Orbit Condition (BOC) If $P$ is an infinite forward orbit of an invertible translation $T$, then $P$ is equal in size to some infinite backward orbit of $T$.

In many contexts, this is a well motivated assumption. The existence of translations with infinite forward and backward orbits is guaranteed among the integers, Euclidean metric spaces, and many other spaces. Where such orbits exist, and totality holds, BOC is not only intuitive but required on pain of arbitrariness: Otherwise we must somehow divide the possible sizes between the forward and backward orbits of $T$, and any way of doing so will be awkward and unmotivated. Furthermore, in Euclidean space, such orbits are rigid rotations or reflections of each other, and thus, like translations, they are exactly alike in structural and qualitative properties. If such similar sets are not equal, then nonequality is arbitrary. We will return to rotation and reflection invariance in the next section. The present point is just that RTI fails under mild conditions even without Discreteness or totality:

REMARK 6.3. Let $T$ be an invertible translation on a metric space $\langle S, d\rangle$ and $P$ an infinite forward orbit of $T$. If $\unlhd$ is Euclidean on $S$ and satisfies BOC then $\unlhd$ violates RTI.

Proof. Let $P=\left\{T^{n} p: n \in \mathbf{N}\right\}$. By BOC, let $P \triangleq R=\left\{T^{-n} q: n \in \mathbf{N}\right\}$ (see Figure 3). Then by PW, $T P \triangleleft P \triangleq R \triangleleft T R$, and hence $T P \triangleleft T R$. So $R \triangleleft P$, but $T R \triangleright T P$, contradicting RTI.

So we do not need to assume Discreteness or totality to get a violation of RTI. Even for Euclidean notions of size that are not notions of discrete count and do not determine all size relations, RTI fails under mild and well motivated assumptions.

When RTI fails, Euclidean size orderings are arbitrary in a clear and robust way: Given one, we can obtain a different but equally valid and equally well (or poorly) motivated one by a mere translation. The point is not that we must accept RTI and therefore reject PW. The point is that if we do not want size relations to be as arbitrary as a choice between positions in a homogeneous coordinate-free space, then we should adopt RTI and abandon PW. 


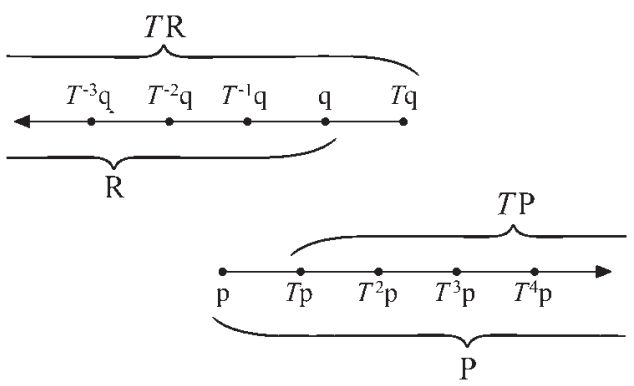

Fig. 3. If $P=\left\{T^{n} p: n \in \mathbf{N}\right\}$ is the same size as $R=\left\{T^{-n} q: n \in \mathbf{N}\right\}$, then PW implies that $T P \triangleleft T R$, so translations do not preserve relative size.

\$7. Rotation invariance. For the same reasons as we would like sizes (absolute and relative) to be translation invariant, we would also like them to be rotation invariant. In many important geometric and physical contexts, there is nothing to distinguish one direction from another, so a notion of size that depends on the particular orientation ${ }^{23}$ of a set is baseless and misleading. But as we will now see, Euclidean sizes must be sensitive to rotation, and very much so.

First, a couple more definitions. For simplicity, we will restrict our attention in this section to Euclidean metric spaces (again, in the geometric sense of Euclidean).

Definition 7.1. A metric space $\langle S, d\rangle$ is Euclidean if for some $n \in \mathbf{N}, S$ is isometric to $\mathbf{R}^{n}$, relative to $d$ and the standard Euclidean metric $\delta(p, q)=\left(\left(p_{1}-q_{1}\right)^{2}+\left(p_{2}-q_{2}\right)^{2}+\right.$ $\left.\cdots+\left(p_{n}-q_{n}\right)^{2}\right)^{1 / 2}$ on $\mathbf{R}^{n}$.

Of course, the existence claims in this section transfer easily to any space that supports rotations and reflections.

We will regard reflections as a subclass of rotations, since a reflection in $n$ dimensions is equivalent to a rotation in $n+1$. Hence, any violation of reflection invariance implies a violation of rotation invariance in a slightly higher-dimensional space, so we might as well lump them together.

\section{DEFINITION 7.2.}

(i) Let $S=\langle S, d\rangle$ be a Euclidean metric space. A map $R: S \rightarrow S$ is a rotation on $S$ if

(a) for some $p \in S$ and all $q \in S, d(p, q)=d(p, R q)$, and

(b) for all $p, q \in S, d(p, q)=d(R p, R q){ }^{24}$

(ii) A transformation $T: A \rightarrow A$ is an involution if for all $p \in A, T T p=p$.

So in particular, reflections and $180^{\circ}$ rotations are both involutions, and both count as rotations here.

Now the principles to be violated:

Absolute Rotation Invariance (ARI) For any rotation $R$ on a Euclidean metric space $\langle S, d\rangle$ and any $A \subseteq S, A \triangleq R A$.

23 We will use 'orientation' in the sense of direction or rotational position rather than handedness. Orientations in this sense are what rotations change.

24 This is a rather limited definition of rotation, but again it is simple and adequate for our purposes. 
Relational Rotation Invariance (RRI) For any rotation $R$ on a Euclidean metric space $\langle S, d\rangle$ and any $A, B \subseteq S, A \unlhd B$ if and only if $R A \unlhd R B$.

ARI simply says that rotations preserve size, and RRI says they preserve size relations.

At first glance, RRI seems weaker than ARI; even if a rotation changes the sizes of sets, could not it always change them in the same way, and thus preserve size relations? But if we assume PW and totality (or weaker, that a set and its reflections are always comparable), then RRI becomes the strongest of all the invariance principles considered here.

REMARK 7.3. Let $S=\langle S, d\rangle$ be a Euclidean metric space and $\unlhd$ a total Euclidean size ordering on $\mathcal{P}(S)$ that satisfies RRI. Then $\unlhd$ also satisfies ARI, ATI, and RTI.

Proof. We first show that involutory rotations preserve size, assuming RRI and totality. Let $R$ be an involutory rotation on $S$. By RRI, $A \unlhd R A$ if and only if $R A \unlhd R R A$. By involution, $R R A=A$, so $A \unlhd R A$ if and only if $R A \unlhd A$. By totality, one of these must hold, so they both do. Thus $A \triangleq R A$, so involutory rotations preserve size.

But any translation or rotation in Euclidean space is a composition of two reflections, and reflections are involutory rotations. Hence, translations and rotations preserve size too, so ARI and ATI hold. RTI follows trivially from ATI. ${ }^{25}$

It follows from this that any total Euclidean size ordering on a geometrically Euclidean space violates ARI and RRI. We already saw (Remark 4.5) that if a translation has an infinite forward orbit, any Euclidean size ordering on the domain of that translation violates ATI. Euclidean metric spaces do have translations with infinite forward orbits, so any total Euclidean size ordering on a Euclidean space violates ATI, and by the proof of Remark 7.3, it also violates ARI and RRI.

For a simple example, consider the sets $\mathbf{N},-\mathbf{N}$, and $\mathbf{- N}-\mathbf{2}=\{-2,-3,-4, \ldots\}$. By $\mathrm{PW},-\mathbf{N}-\mathbf{2} \triangleleft \mathbf{- N}$, but both are rotations of $\mathbf{N}$. (Even if we exclude reflections, they are still rotations of $\mathbf{N}$ in the complex plane.) So rotations do not always preserve size. And since $\mathbf{N}$ cannot be equal in size to both $-\mathbf{N}$ and $-\mathbf{N}-\mathbf{2} \triangleleft-\mathbf{N}$, totality implies that either $\mathbf{N} \triangleleft-\mathbf{N}$ or $-\mathbf{N}-\mathbf{2} \triangleleft \mathbf{N} .{ }^{26}$ In either case we have a set $A$ (either $\mathbf{N}$ or $-\mathbf{N}-\mathbf{2}$ ) and a rotation $R$ such that $A \unlhd R A$ but not $R A \unlhd R R A$, contradicting RRI. So rotations cannot always preserve relative sizes either, given PW and totality (or PW and comparability of reflections).

One of the interesting things about rotation invariance is that it contradicts PW even for sets confined to a small bounded region. ${ }^{27}$

Definition 7.4. Given a metric space $\langle S, d\rangle$, a set $A \subseteq S$ is bounded if there is some $n \in \mathbf{N}$ such that for all $p, q \in A, d(p, q)<n$.

The fact that translations do not preserve Euclidean sizes is pretty obvious if we consider unbounded sets like $P$ and $T P$ above, or half-intervals like $(0, \infty)$ and $(1, \infty)$, half-planes, and so on. It is also well known that finitely additive measures (such as numerosities) violate rigid transformation invariance for bounded sets, in virtue of the very sophisticated

25 If we exclude reflections from RRI and let it apply only to handedness-preserving rotations we can still obtain $\mathrm{RRI} \Rightarrow \mathrm{ATI} \Rightarrow \mathrm{RTI}$ and $\mathrm{ARI} \Rightarrow \mathrm{ATI} \Rightarrow \mathrm{RTI}$, since $180^{\circ}$ rotations are also involutions, and every translation is a composition of two $180^{\circ}$ rotations. However, not all rotations are compositions of $180^{\circ}$ handedness-preserving rotations within a given space, so ARI does not follow from such a weakened RRI.

26 This is because $\mathbf{N} \unlhd-\mathbf{N}-\mathbf{1}$ or $-\mathbf{N}-\mathbf{1} \unlhd \mathbf{N}$, where $-\mathbf{N}-\mathbf{1}=\{-n-1: n \in \mathbf{N}\}$.

27 This is also true for translation invariance in some non-Euclidean spaces, for example, tori, where some translations are effectively rotations. 


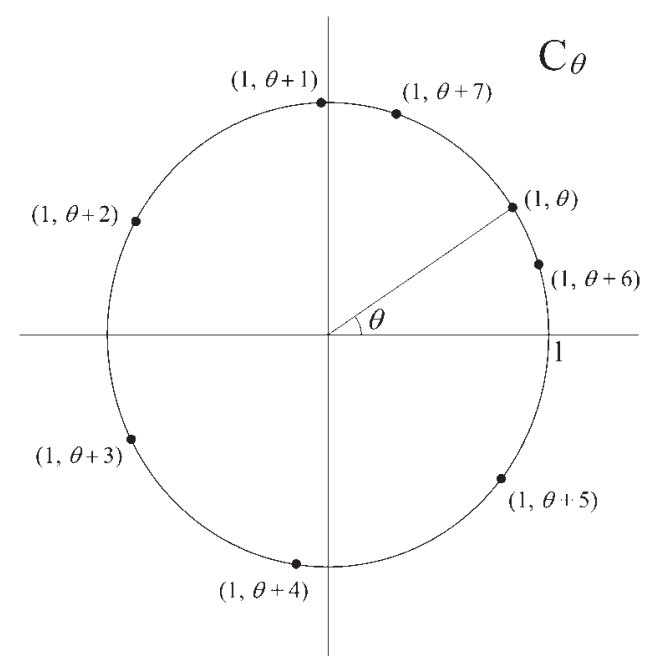

Fig. 4. The first eight points in $C_{\theta}=\{(1, \theta+n): n \in \mathbf{N}\}$ for an arbitrary angle $\theta$. Note $C_{\theta+m} \subset C_{\theta} \subset C_{\theta-m}$ for $m \in \mathbf{N}$, and the angles $\theta \pm m$ are dense in the circle.

Banach-Tarski paradox. But it is worth noting that Euclidean sizes in general, whether additive or not, must violate rotation invariance even for very simple bounded sets-much simpler than the Banach-Tarski partitioning of the sphere.

In fact we can see this with a countable bounded point set in the plane. All we need is a nonperiodic rotation. For example, consider the points $(1, \theta)$ on the unit circle, written in polar coordinates with the angle $\theta$ in radians. Let $R(1, \theta)=(1, \theta+1)$, a rotation of one radian. Since this is incommensurable with the full revolution of $2 \pi$ radians, the points $R^{n} p$ and $R^{m} p$ will never coincide for $n \neq m$. Hence $R$ has an infinite forward orbit $C_{\theta}=\left\{R^{n}(1, \theta): n \in \mathbf{N}\right\}$ (see Figure 4). By Lemma 4.4, $R C_{\theta} \triangleleft C_{\theta}$ for any size ordering $\unlhd$ that is Euclidean on the subsets of $C_{\theta} \cdot{ }^{28}$ So we get the following:

REMARK 7.5. Let $\langle S, d\rangle$ be a Euclidean metric space of at least two dimensions ${ }^{29}$ and let $\unlhd$ be Euclidean on $\mathcal{P}(S)$. Then $\unlhd$ violates $A R I$, and if $\unlhd$ is total on $\mathcal{P}(S)$, it also violates RRI.

Proof. The example of $C_{\theta}$ and $R C_{\theta}$ is enough to show that $\unlhd$ violates ARI. If $\unlhd$ is total, it also violates RRI by Remark 7.3

But more than the trivial result, I want to emphasize the example of $C_{\theta}$ and $R C_{\theta}$ : simple, countable, bounded sets for which rotation invariance must fail. Given PW-even without Discreteness or finite additivity-simple bounded sets that are mere rotations of

28 This example is closely related to a puzzle that Frank Arntzenius once gave me: find a set of points on a sphere such that one can obtain a proper subset just by rotating the sphere. The example also shows that Common Notions 4 and 5 of the Elements are incompatible even for bounded figures. See footnote 18.

29 In fact, the preceding example of of $\mathbf{N}$ and $\mathbf{- N}-\mathbf{2}$ shows that this holds in one dimension as well. If we exclude reflections and restrict RRI to the handedness-preserving rotations within a given space, then RRI may be consistent with PW in one-dimensional spaces, but not two, since a reflection in one dimension is a rotation in two. And in any case, such a weakened RRI is still not consistent with PW and Discreteness, which imply that the size relation between $\mathbf{N}$ and $\mathbf{N}+\mathbf{1} / \mathbf{2}=\{n+1 / 2: n \in \mathbf{N}\}$ differs from that between $\mathbf{N}+\mathbf{1} / \mathbf{2}$ and $\mathbf{N}+\mathbf{1}$. 
each other must differ in size, and as well the size relation between two sets depends on their particular orientations, even while their orientations to each other remain fixed. This is again deceptive, for attributing different sizes to two sets suggests a substantive difference, while under PW it may merely reflect a difference in direction. In contexts where there is no privileged direction or coordinate system, this means that both absolute and relative set sizes are as arbitrary as a choice of coordinate axes.

As with RTI, we can also get a violation of RRI without Discreteness or totality. I will not go through the details; they are essentially the same as for RTI. All we need is a version of the Backward Orbit Condition for rotations, which is straightforward.

But Euclidean sizes not only vary with rotation, they are radically sensitive to it: bounded sets that differ only by an arbitrarily small rotation nonetheless differ in size by arbitrarily large finite quantities, in the sense that the set difference between two arbitrarily near sets $C_{\theta}$ and $C_{\rho}$ has arbitrarily large finite power:

REMARK 7.6. For any $n \in \mathbf{N}$ and $\theta \in[0,2 \pi)$ there are angles $\rho$ and $\sigma$ arbitrarily close to $\theta$ such that $C_{\rho} \subset C_{\theta} \subset C_{\sigma}$ and $\left|C_{\theta} \backslash C_{\rho}\right|,\left|C_{\sigma} \backslash C_{\theta}\right|>n$.

Proof. The angles $\theta+m$ for $m \in \mathbf{N}$ are dense in the circle, so choose $m>n$ such that $\theta+m$ is arbitrarily close to $\theta$. Then $\left|C_{\theta} \backslash C_{\theta+m}\right|=\mid\{(1, \theta),(1, \theta+1), \ldots$, $(1, \theta+m-1)\} \mid=m>n$. Likewise choose $k>n$ such that $\theta-k$ is arbitrarily close to $\theta$. Then $\left|C_{\theta-k} \backslash C_{\theta}\right|=k>n$.

So there are angles $\rho$ and $\sigma$ arbitrarily close to $\theta$ such that $C_{\theta}$ contains all of $C_{\rho}$ as well as an arbitrarily large finite number of other points, and $C_{\sigma}$ contains $C_{\theta}$ and an arbitrarily large finite number of other points. These are simple facts of geometry and set theory independent of PW or any other postulates about infinite sizes (since $C_{\theta+i} \backslash C_{\theta+j}$ is finite). But under PW, it makes sense to say that $C_{\theta-k}$ is larger than $C_{\theta}$ by $k$ elements, since $C_{\theta} \triangleleft C_{\theta-1} \triangleleft C_{\theta-2} \triangleleft \ldots \triangleleft C_{\theta-k}$. So in this sense, arbitrarily small rotations cause arbitrarily large finite changes in size. ${ }^{30}$

In a sense this is unsurprising. After all, continuous, unbounded sets are even more sensitive to small rigid transformations. The set-difference between the intervals $(x, \infty)$ and $(x+\epsilon, \infty)$, for arbitrarily small $\epsilon$, is uncountably infinite. But the example of the sets $C_{\theta}$ is worse. There we have bounded, countable sets whose Euclidean sizes vary both up and down with tiny rotations in the same direction. Turn $C_{\theta}$ a hair clockwise and it becomes a thousand points larger. Turn it another hair clockwise and it becomes a billion points smaller.

This sensitivity amplifies the fundamental arbitrariness and limitations of utility implied by the failure of absolute rotation invariance. Not only do Euclidean sizes depend on an arbitrary choice of coordinate axes, they depend so sensitively on it that the tiniest difference in direction can make a vast difference in size. Similarity and proximity are not even good guides to approximate size, if PW holds. Even in spaces where there is no preferred direction or designated coordinate system, Euclidean set sizes depend so sensitively on direction that indiscernible differences of angle imply size differences larger than any finite number one can fully comprehend. In this respect, Euclidean size is an awkward and misleading way of classifying sets.

30 For the numerosities of Benci et al., which are additive and include the whole numbers, we can say more concretely that for any $n \in \mathbf{N}$, there are arbitrarily small rotations $R$ such that $\left[R C_{\theta}\right]>\left[C_{\theta}\right]+n$. 
§8. Conventionalist pluralism. The central problem with Euclidean sizes is arbitrariness. Where disjoint infinite sets are concerned, there is no apparent motivation for assigning one size relation rather than another, ${ }^{31}$ and to choose one often involves privileging an arbitrary position or direction. One might take a pluralistic view of this situation and respond as follows: the size of a set is naturally going to depend on what one means by 'size.' There are multiple theories available, including Cantor's theory and many Euclidean theories, which differ in the sizes and size relations they assign to particular sets. So yes, says the pluralist, set sizes are indeterminate until one chooses a sufficiently strong concept or theory of size, but that is no surprise. We can define size in many different ways, even assuming PW, and this is an advantage. It means we have many Euclidean size functions at our disposal to use for different purposes.

It should be clear from the introduction that I am sympathetic to this sort of pluralism in general, but there are problems with the view just stated. Firstly, we do not really have a Euclidean theory or concept of set size that determines the sizes of the simple sets discussed above. The theory of Benci et al. shows that Euclidean assignments of size exist for large classes of sets (assuming the existence of selective ultrafilters), but we have drawn a distinction between assignments and theories (\$2). An assignment is any function or relation, however complex, noncomputable, or undefinable, while a theory must be somewhat comprehensible and communicable for the sake of usefulness. Likewise, a concept must be something we can grasp, at least in a piecemeal way, if the word is to support its psychological uses. But there is no reason to think that a strong and broad Euclidean size assignment can be determined by any tractable theory or intelligible concept. As noted in $\S 6$, we so far have no explicit definition of a particular Euclidean size assignment on the subsets of $\mathbf{N}$ or of any infinite set. All known existence proofs for such assignments rely on nonconstructive axioms such as Choice and Martin's Axiom. While this does not prove that such assignments cannot be explicitly constructed or uniquely defined, it gives us reason to suspect that is the case. And if so, then we are not just facing an arbitrary choice between different notions of size; there is nothing as useful as a Euclidean notion of size that determines the sizes of sets like those discussed above. So pluralists may be right that it is useful in principle to have a variety of different size measures at hand, but they cannot refer to Euclidean assignments as theories or concepts without risk of misleading. Nor can they take it for granted that the differences between Euclidean assignments are really useful, given that they might not even be expressible.

Secondly (against the above-mooted pluralist position), we have seen examples of sets such that any Euclidean assignment that determines their sizes will incorporate unmotivated details. Such assignments are misleading, for while some of the sizes they assign might reflect logical facts or consequences of independently interesting theories (like ZFC), others will be purely arbitrary, so that it becomes difficult to tell which results have significance and which do not. Having a variety of conceptual tools at one's disposal may be a benefit, but if each of these tools mixes meaningful content with arbitrary stipulations in a way that's difficult to extricate, that's detrimental.

31 At least for infinite sets of the same power. One might suppose that the size relations between sets with different powers are determined by their powers: greater power is sufficient for greater size, but not necessary. Benci et al. call this the Half-Cantor Principle (Benci et al., 2006, 2007), and it is consistent with PW. The difficulty lies in fixing the size relations between infinite sets of the same power. 
So the pluralist view is correct in that a theory of size need not be a theory of the one true notion of size and its properties, but a useful theory is one that conveys factual or logical insights of some kind, and preferably does not mix these with unmotivated and inexpressibly complex choices.

\$9. Partial size assignments. Since Euclidean set sizes are not in general determined by well motivated principles (nor perhaps by comprehensible ones), one solution might be to adopt a partial Euclidean size assignment as a theory of size. On this approach, for example, Even might be regarded as neither smaller than Odd, nor larger, nor equal. Consider the theory consisting of just PW, the Half-Cantor Principle of Benci et al. (2006, 2007) (namely that if $|A|<|B|$ then $A \triangleleft B$ ), and the stipulation that where these two postulates do not determine relative size, no size relation holds. So here sets of larger power are larger and proper subsets are smaller, but sets of the same power such that neither is contained in the other have no definite size relation. Would this not be better than Galileo's solution (1939), according to which no infinite sets have relative sizes? And would it not be in some ways better than Cantor's, which fails to discriminate at all between sets of the same power, even when one contains the other?

One possible objection to this proposal is that it adds nothing to the familiar concepts of power and proper subset. In fact it obscures those concepts a little. To say that $A$ is smaller than $B$, in this theory, is just to say that either $A$ has smaller power than $B$ or $A$ is a proper subset of $B$ (or both). Is it not better to distinguish these cases, just as we already do in our standard language of set theory? Uniting the relations of proper subset and smaller power in one relation muddies things. But if we bring in further principles, such as Discreteness, RTI (which appears to be consistent with PW if we do not assume totality or the Backward Orbit Condition), or algebraic properties like those proposed by Benci et al., the union may not be so fruitless. It is very doubtful that any combination of such properties will determine all Euclidean size relations, but perhaps some combination could yield a partial Euclidean assignment that is both comprehensible and enlightening in applications.

That said, even partial Euclidean assignments must suffer some arbitrariness. Whatever further properties we may add, PW alone implies that size is not preserved by rigid transformations, even for bounded point sets. The relative sizes of $P$ and $T P$, for example, or $C_{\theta}$ and $R C_{\theta}$, are determined by PW, since in each case the first set includes the second. Even disjoint sets like $Q$ and $T Q$ that are as alike as can be cannot always have the same size. This means that the sizes of qualitatively identical sets depend on their particular positions in space, which is clearly arbitrary, especially in contexts where there is no privileged origin or direction. Further, size must vary radically under arbitrarily small rotations, whether or not totality holds, for the relations between arbitrarily near sets $C_{\theta}$ and $C_{\theta+i}$ are determined by PW alone. All of this follows directly from PW without assuming totality, so abandoning totality will not save us from arbitrariness.

§10. The underdetermination of powers. One last objection must be answered. I have argued that Euclidean assignments and theories must be either largely arbitrary or very weak - too weak to decide simple cases like $Q$ and $T Q$. But the standard Cantorian theory of set size is also indeterminate, in virtue of the independence of the Continuum Hypothesis $(\mathrm{CH})$ from ZFC, and other such independence results. Cantor's theory does not 
even tell us whether the size of the continuum is the second infinite power, so how are Euclidean theories any worse ? $^{32}$

I do not want this discussion to degenerate into a contest between Cantorian and Euclidean theories; the limitations of Euclidean theories are what they are regardless of how Cantor's theory fares. But there is a difference worth noting between the indeterminacy of Cantorian sizes and that of Euclidean sizes. Cantorian sizes are indeterminate because the underlying set theory is, independently of any notion of size. $\mathrm{CH}$, though it is often stated informally in terms of size, reduces to a proposition about one-to-one correspondence. It says that the continuum (or $\mathcal{P}(\mathbf{N})$ ) has a one-to-one correspondence with a subset of each infinite set that does not have a one-to-one correspondence with $\mathbf{N}$. ZFC does not determine whether that statement is true. But once the truth or falsity of that statement is stipulated, the Cantorian size relation between the continuum and sets of the second infinite power is thereby fixed. So the indeterminacy of Cantorian sizes lies not in the Cantorian notion of size itself, but in the incompleteness of the underlying size-free set theory.

Not so for Euclidean sizes. If every proposition expressible in the language of ZFC were determined, this would still not fix the Euclidean sizes of sets, for we have no rule for assigning sizes to sets based on their set-theoretic properties. As we have seen above, neither the relation of bijection nor even isometry is enough to determine Euclidean size relations. To put it another way, we at least know what power consists in: one-to-one correspondence. But what constitutes Euclidean size is only partly specified; it is a mixture of common sense (PW) and chimera.

\$11. Conclusion. It would be unfortunate if the observations made here had any negative impact on research programs like that of Benci et al. Such work has shown us that Euclidean size assignments are logically consistent, and Euclidean conceptions of size need not be mere misconceptions. As well it has revealed the subtle conditions under which such assignments exist, and their connections with other deep and interesting topics. And perhaps there is some hope for partial Euclidean size assignments that are comprehensible and enlightening, even if some of their consequences are uninformative or misleading.

But the problems of arbitrariness that plague Euclidean assignments are severe. Our examples show that any Euclidean theory or assignment that is strong and broad enough to relate even simple, countable sets like those discussed above will be arbitrary and misleading. Furthermore, this is no mere technicality engendered by particular devices such as the Axiom of Choice or selective ultrafilters. It is a clear, fundamental constraint on any application of PW to infinite sets, including simple sets of whole numbers and even bounded, countable point sets. Euclidean theories and assignments go a long way as illustrations of mathematical freedom and interesting connections within mathematics, but as theories of size in themselves they are deeply and disappointingly limited. The problem is not that Euclidean theories are false or counterintuitive. It is that they are either very weak and narrow or arbitrary and misleading.

Acknowledgments. I would like to thank Sylvia Wenmackers, Paolo Mancosu, Leon Horsten, Tim Button, Foad Dizadji-Bahmani, Frank Arntzenius, and Jeremy Gwiazda for

32 Thanks to Paolo Mancosu and Leon Horsten for raising this question. 
helpful comments on earlier drafts, and the University of Bristol and the Centre for Philosophy of Natural and Social Science for research support.

\section{BIBLIOGRAPHY}

Benci, V., \& Di Nasso, M. (2003a). Numerosities of labeled sets: A new way of counting. Advances in Mathematics, 173, 50-65.

Benci, V., \& Di Nasso, M. (2003b). Alpha-theory: An elementary axiomatics for nonstandard analysis. Expositiones Mathematicae, 21, 355-386.

Benci, V., Di Nasso, M., \& Forti, M. (2006). An Aristotelean notion of size. Annals of Pure and Applied Logic, 143, 43-53.

Benci, V., Di Nasso, M., \& Forti, M. (2007). An Euclidean measure of size for mathematical universes. Logique et Analyse, 50, 43-62.

Benci, V., Horsten, L., \& Wenmackers, S. (2013). Non-Archimedean probability. Milan Journal of Mathematics, 81, 121-151.

Blass, A., Di Nasso, M., \& Forti, M. (2012). Quasi-selective ultrafilters and asymptotic numerosities. Advances in Mathematics, 231, 1462-1486.

Bolzano, B. (1950). Paradoxes of the Infinite; transl. by Prihonsky, F., London: Routledge.

Bolzano, B. (1973). Theory of Science; Transl. by Terrell, B., edited by Berg, J., Dordrecht, The Netherlands: D. Reidel Publishing.

Bunn, R. (1977). Quantitative relations between infinite sets. Annals of Science, 34, 177-191.

Di Nasso, M. (2010). Fine asymptotic densities for sets of natural numbers. Proceedings of the American Mathematical Society, 138, 2657-2665.

Di Nasso, M., \& Forti, M. (2010). Numerosities of point sets over the real line. Transactions of the American Mathematical Society, 362, 5355-5371.

Galileo (1939). Dialogues Concerning Two New Sciences. Evanston, IL: Northwestern University. Reprinted by Dover 1954.

Gödel, K. (1947). What is Cantor's continuum problem? American Mathematical Monthly, 54, 515-525.

Gwiazda, J. (2010). Probability, hyperreals, asymptotic density, and God's lottery. PhilSci Archive. Available from: http://philsci-archive.pitt.edu/id/eprint/5527.

Heath, T. (1956). The Thirteen Books of Euclid's Elements. New York: Dover.

Katz, F. M. (1981). Sets and their sizes. PhD Thesis, MIT. Available from: http://citeseerx.ist.psu.edu/viewdoc/summary?doi= 10.1.1.28.7026.

Leibniz, G. W. (2001). The Labyrinth of the Continuum: Writings on the Continuum Problem, 1672-1686. R. T. W. Arthur, translator and editor. New Haven, CT: Yale University Press.

Mancosu, P. (2009). Measuring the size of infinite collections of natural numbers: Was Cantor's theory of infinite number inevitable? Review of Symbolic Logic, 2, 612-646.

Mayberry, J. (2000). The Foundations of Mathematics in the Theory of Sets. Cambridge: Cambridge University Press.

McCall, S., \& Armstrong, D. M. (1989). God's lottery. Analysis, 49, 223-224.

Parker, M. W. (2009). Philosophical method and Galileo's paradox of infinity. In van Kerkhove, B., editor. New Perspectives on Mathematical Practices. Hackensack, NJ: World Scientific, pp. 76-113.

Pincus, D., \& Solovay, R. (1977). Definability of measures and ultrafilters. Journal of Symbolic Logic, 42, 179-190.

Resnik, M. (1997). Mathematics as a Science of Patterns. Oxford: Clarendon Press. 
Tannery, P. (1884). Sur l'authenticité des axioms d'Euclide. Bulletin des sciences mathématiques et astronomiques, 2e série, 8, 162-175.

Wenmackers, S., \& Horsten, L. (2013). Fair infinite lotteries. Synthese, 190, 37-61.

Williamson, T. (2007). How probable is an infinite series of heads? Analysis, 67, 173-180.

Wimsatt, W. C. (1981). Robustness, reliability, and overdetermination. In Brewer, M. B., and Collins, B. E., editors. Scientific Inquiry and the Social Sciences. San Francisco, CA: Jossey Bass, pp. 124-163. Reprinted in W. C. Wimsatt, 2007, Re-engineering Philosophy for Limited Beings, pp. 43-71.

CENTRE FOR PHILOSOPHY OF NATURAL AND SOCIAL SCIENCE

LONDON SCHOOL OF ECONOMICS AND POLITICAL SCIENCE

HOUGHTON STREET, LONDON WC2A 2AE, UK

E-mail:m.parker@1se.ac.uk 